\title{
Bilateral ovarian serous cystadenocarcinoma metastasizing to cervix: a rare case report
}

\author{
Arati Mallick ${ }^{1}$, Saubhagya K. Jena ${ }^{1 *}$, Debasis Kuanar ${ }^{2}$ \\ ${ }^{1}$ Department of Obstetrics and Gynecology, All India Institute of Medical Sciences, Sijua, Bhubaneswar, Odisha, India \\ ${ }^{2}$ Department of Anesthesiology, Care Hospital, Bhubaneswar, Odisha, India
}

Received: 22 July 2018

Accepted: 14 September 2018

\section{*Correspondence:}

Dr. Saubhagya K. Jena,

E-mail: drsaubhagya@gmail.com

Copyright: (C) the author(s), publisher and licensee Medip Academy. This is an open-access article distributed under the terms of the Creative Commons Attribution Non-Commercial License, which permits unrestricted non-commercial use, distribution, and reproduction in any medium, provided the original work is properly cited.

\begin{abstract}
Ovarian carcinoma is the second most common gynaecologic cancer and the leading cause of death from gynaecologic malignancy. Two-third of all malignant epithelial ovarian tumors are constituted by serous ovarian cystadenocarcinomas. It is generally observed that ovarian cancer tends to remain intraabdominal even in advanced cases and that dissemination is usually by invasion of adjacent viscera, diffuse intraperitoneal implantation, and metastatic involvement of aortic and pelvic lymph nodes. Metastasizes to the uterine cervix, vagina, or vulva in ovarian cancer is rare. The reverse i.e. ovarian metastasis from cervical tumor is rather more common. Published literature suggest that, patients with cervical metastases had associated malignant ascites, retroperitoneal lymph node involvement, and significant peritoneal carcinomatosis. Cervical metastasis in ovarian malignancies always indicates the advanced stage of tumor and multi-organ involvement, indirectly stating poor prognosis. The median survival in cases of ovarian cancer metastasizing to cervix is 4.4 months. Authors report a case of bilateral ovarian serous cystadenocarcinoma metastasising to posterior lip of cervix resulted in poor prognosis and proved fatal for the patient with review of published literature.
\end{abstract}

Keywords: Cervical metastasis, Ovarian cancer, Ovarian serous cystadenocarcinoma, Secondary tumors in uterine cervix

\section{INTRODUCTION}

Ovarian carcinoma is the second most common gynaecologic cancer and the leading cause of death from gynaecologic malignancy. Two-third of all malignant epithelial ovarian tumors are constituted by serous ovarian cystadenocarcinomas. Ovarian cancer usually metastasizes to peritoneum, intestinal surfaces, bladder, visceras as lung and liver, lymph nodes.

Occurrence of cervical metastasis from ovarian tumor is very unusual and rare, the reverse i.e. ovarian metastasis from cervical tumor is rather more common. In the same way metastasis from ovarian cancer to vagina and/or vulva also very rarely detected. ${ }^{1,2}$ Most common mode of metastasis in secondary cervical involvement is usually, direct spread of endometrial carcinoma to the cervix. ${ }^{3}$ But hematogenous spread of tumor emboli leading to cervical metastasis has been detected in cases of ovarian malignancy and cancer of stomach, gall bladder, pancreas, breast and lung. ${ }^{4-7}$ Cervical metastasis in the above malignancies always indicates the advanced stage of tumor and multiorgan involvement, indirectly stating poor prognosis.

In a review of 148 patients with FIGO Stage III or IV ovarian cancer, 7 patients had cervical metastatic deposits. Four were due to direct extension of the primary; the 3 patients who had truly metastatic deposits, all presented with cervical cytology showing the presence 
of adenocarcinoma cells. The median survival in cases of ovarian cancer metastasizing to cervix is 4.4 months. $^{8}$ Hence, multimodal aggressive approach is needed, good collaboration between gynaecologist, other clinicians, and pathologist as well as histopathology and immunohistochemical features of tumor is needed to arrive at accurate diagnosis. ${ }^{9}$ Herein authors report a case of Bilateral ovarian serous cystadenocarcinoma metastasising to posterior lip of cervix resulted in poor prognosis and proved fatal for the patient.

\section{CASE REPORT}

A fifty-two-year, postmenopausal lady presented with abdominal distension and shortness of breath for one month, associated with loss of appetite and weight. She attained menopause three years back. Her previous menstrual cycles were regular with normal flow. She had four full term vaginal deliveries before and her last child birth was 20 years back. She was not sterilized. Her past and family history was insignificant. On examination patient was cachectic, had mild pallor. Vitals were stable, except decreased breath sounds in both side of chest suggestive of bilateral pleural effusion. Bilateral breasts were soft and normal. Abdomen was distended, with tense ascites. Omental thickening around umbilicus was felt. Per speculum examination showed healthy and high up cervix. In bimanual pelvic examination uterus size could not be assessed due to ascites and all fornices were full.

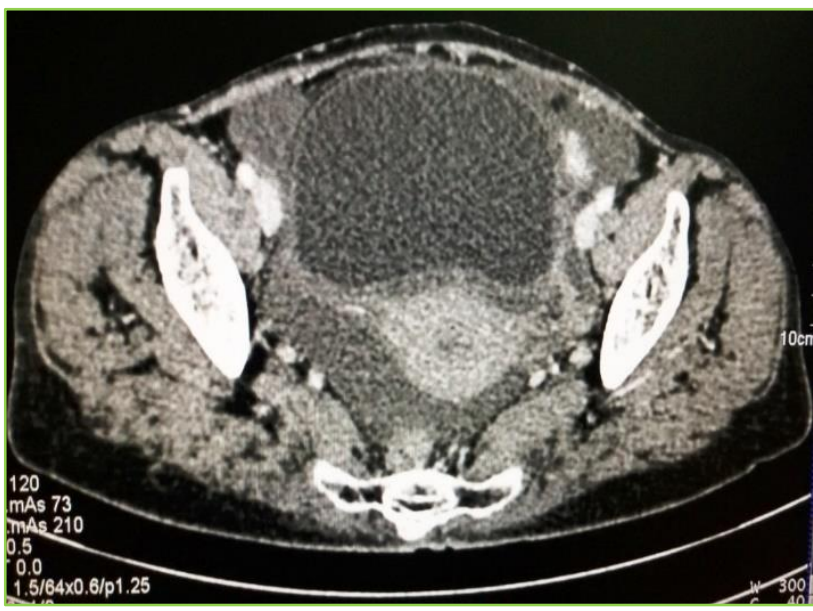

Figure 1: Contrast enhanced computed tomography (CECT) image showing left adnexal mass.

Ultrasonography showed thickened omentum with gross ascites. Left ovary was enlarged with papillary growth of size $6 \times 5 \times 4 \mathrm{~cm}^{3}$. Contrast enhanced computed tomography (CECT) revealed bilateral pleural effusion (left>right), gross ascites with peritoneal thickening and omental thickening. Left adnexal mass (solid and cystic) of size $6 \times 5 \times 4 \mathrm{~cm}^{3}$. Serum CA 125 was elevated to 3197 $\mathrm{U} / \mathrm{ml}$. Peritoneal fluid cytology was positive for malignancy. Stool for occult blood was negative. Both Upper GI Endoscopy and Colonoscopy were normal.
Patient was planned for NACT (Neo Adjuvant Chemo Therapy) because of her gross ascites, bilateral pleural effusion and poor condition. She received five cycles of neoadjuvant chemotherapy at three-week interval each with Paclitaxel (220mg) and Carboplatin (400mg). Post chemotherapy CECT showed enhancing soft tissue mass of size $4 \times 3 \mathrm{~cm}^{2}$ (both solid and cystic) in left adnexa suggestive of ovarian mass. Right ovary normal measuring $2.5 \times 3 \mathrm{~cm}^{2}$. Uterus was normal, thickened omentum, moderate ascites with peritoneal enhancing thickening. No pleural effusion and no lymphadenopathy (Figure 1).

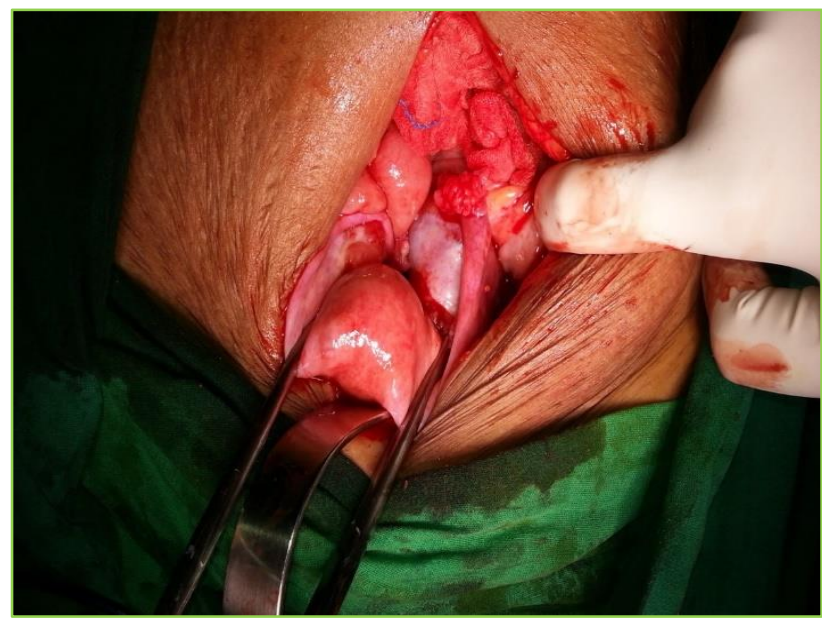

Figure 2: Intraoperative image showing left ovarian tumor with grossly normal uterus and right ovary.

Hence Exploratory laparotomy with cytoreductive surgery was planned after $5^{\text {th }}$ cycle of chemotherapy. On exploratory laparotomy, $50 \mathrm{ml}$ of hemorrhagic ascitic fluid was drained. Uterus and right ovary grossly normal. Left ovarian tumour $5 \times 4 \mathrm{~cm}^{2}$ adhered to nearby structures (Figure 2), with lots of metastatic deposits over peritoneum, omentum, under surface of diaphragm. Large omental cake was present, adherent to large bowel (Figure 3).

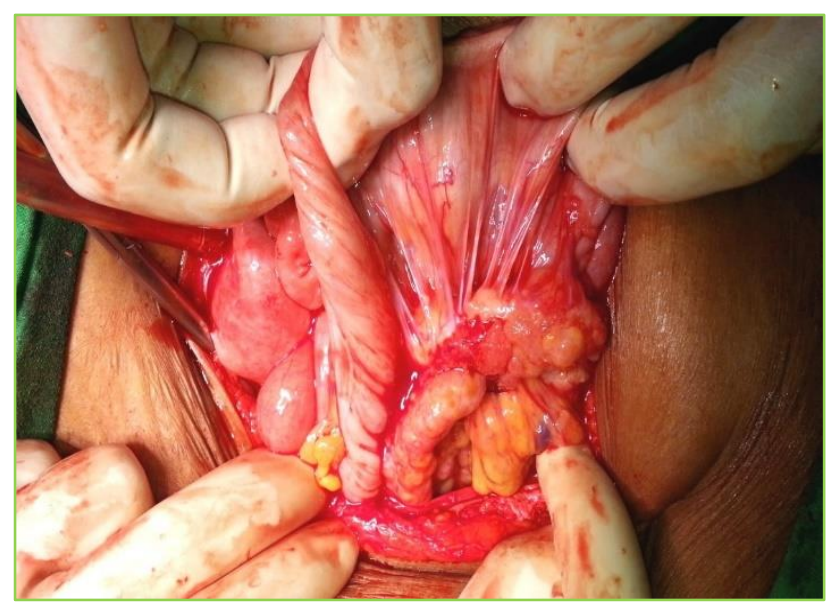

Figure 3: Intraoperative image showing large omental cake. 


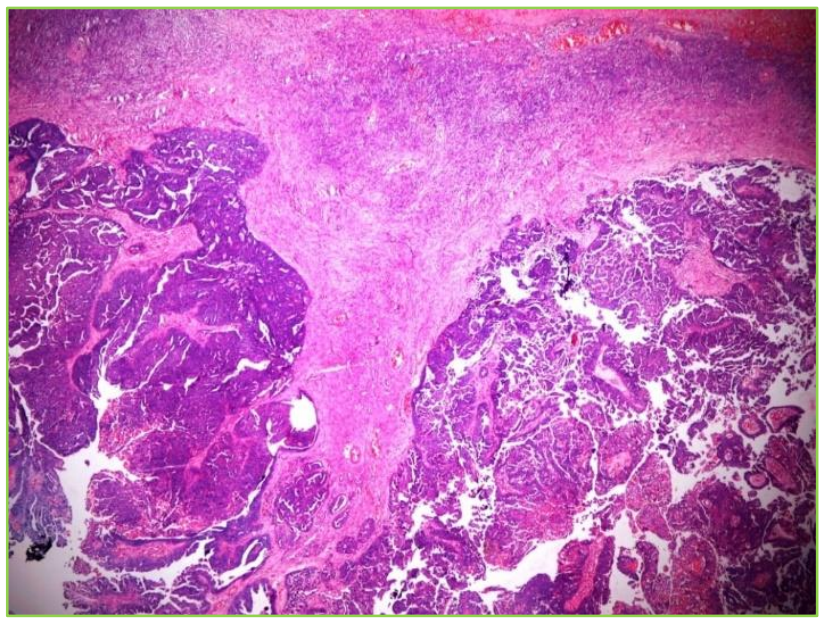

Figure 4: Histological image of left ovary showing papillary growth $(\mathrm{H}$ and $\mathrm{E}$ 100x).

Total abdominal hysterectomy with bilateral salpingoophorectomy was performed along with infracolic omentectomy and resection of peritoneal lesions. No enlarged retroperitoneal lymph nodes were palpated during surgery. Grossly specimen showed, bilateral ovarian surface was bosselated, with papillary excrescence and breach in capsule. Both ovaries were enlarged with left ovary measuring $4.8 \times 2.8 \times 3 \mathrm{~cm}^{3}$ and right ovary measuring $3.2 \times 1.8 \times 1.6 \mathrm{~cm}^{3}$. Cut section showed bilateral ovaries having both solid and cystic components. Lumen of both fallopian tubes were obliterated. Endometrium was atrophied. Myometrium and cervix was unremarkable. Broad ligament contained a fungating papillary growth $(0.8 \mathrm{~cm})$. Histopathology showed primary papillary serous cystadenocarcinoma involving both right and left ovaries (Figure4). Both fallopian tubes including fimbrial ends were involved (Figure 5). Both side parametrium, broad ligament, omentum and peritoneal surface showed diffuse involvement by tumour.

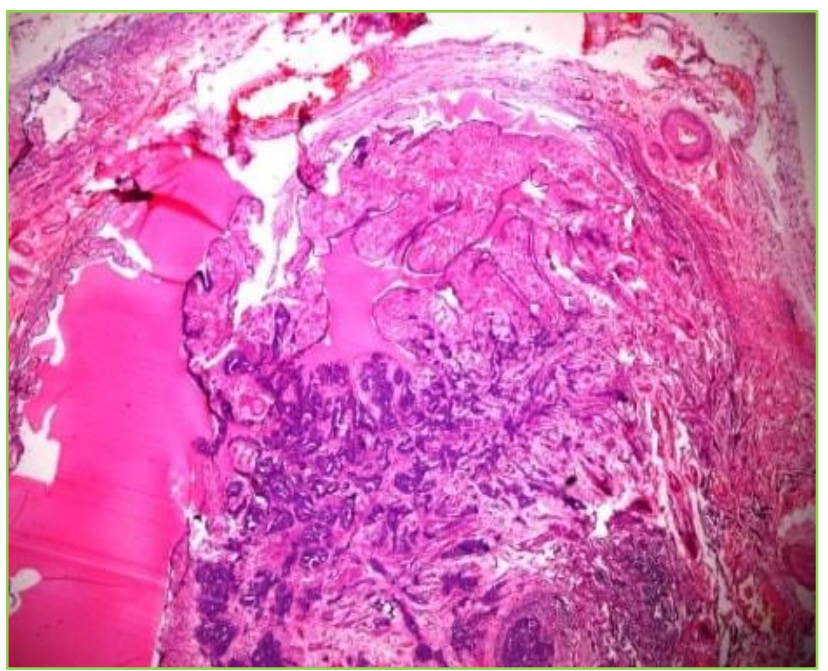

Figure 5: Histological image of tube showing tumor implants (H and E 100x).

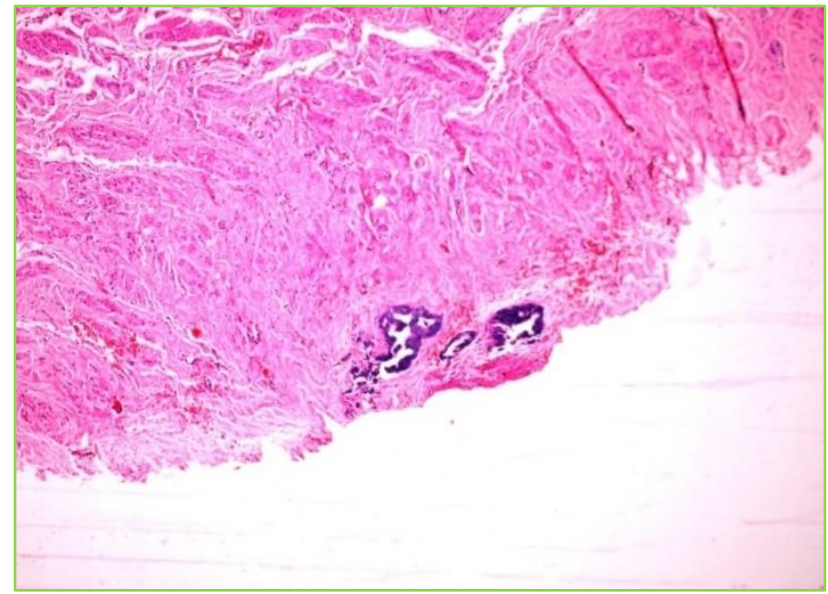

Figure 6: Histological image of tumour deposit in posterior lip of cervix in low power $(\mathrm{H}$ and $\mathrm{E}$ 10x).

Outer surface of posterior lip of cervix showed tumour deposit, suggesting cervical metastasis. Immunohistochemistry showed tumour and cervical implant, both positive for CK7, CA125, ER indicating an ovarian primary origin of tumor and secondary cervical metastasis (Figure 6 and 7).

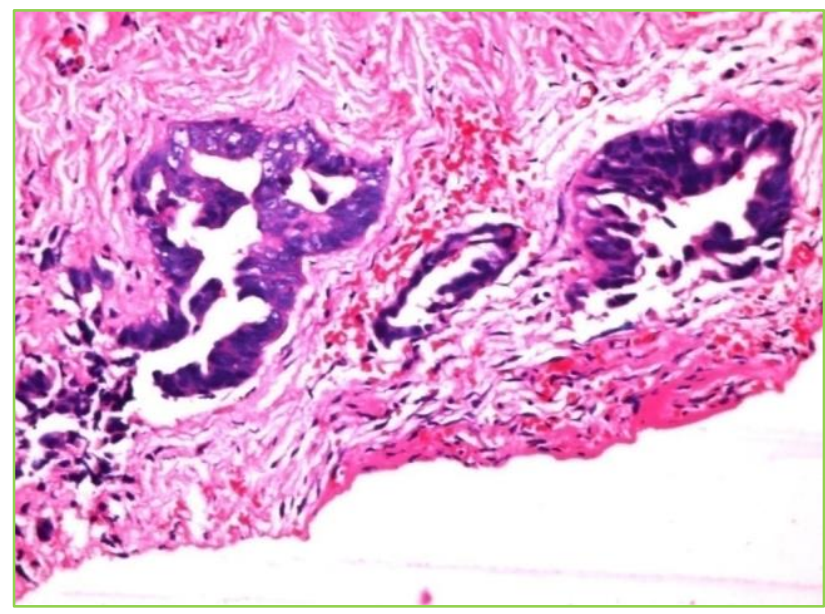

Figure 7: Histological image of tumour deposit in posterior lip of cervix in high power ( $\mathrm{H}$ and $\mathbf{E} \mathbf{1 0 x})$.

Her postoperative period was uneventful. Stiches were removed on $10^{\text {th }}$ postoperative day and she was discharged well. Patient received 4 cycles of postoperative chemotherapy with carboplatin and paclitaxel and was on proper follow-up. But cervical metastasis proved fatal for the patient and authors lost her 11 months after surgery, irrespective of proper treatment.

\section{DISCUSSION}

Almost invariably $50-80 \%$ of all malignant epithelial ovarian tumours are constituted by Serous ovarian cystadenocarcinomas. Omental metastasis is common resulting in gross ascites in these tumors. Ovarian cancer usually metastasizes to peritoneum, intestinal surfaces, 
bladder, visceras as lung and liver, lymph nodes. ${ }^{10}$ These tumors were usually superficially invasive and limited within peritoneal cavity, rarely metastasize to distant places as suggested by many retrospective clinical studies. ${ }^{10,11}$

Metastasis of ovarian carcinoma occurs by direct extension from the ovarian tumour to neighbouring organs such as bladder, colon or when cancer cells detach from the primary tumour, meets peritoneal fluid and disseminated to peritoneum, whole of abdominal cavity. In advanced, high-grade serous carcinomas, extensive peritoneal seedling by tumor cells leads to gross ascites. Rapid growth, early metastasis and aggressive disease course is a feature of these tumors. Hematogenous metastasis is uncommon in case of ovarian tumor, but lymphatic metastasis to pelvic and/or para-aortic lymph nodes can occur in some cases. ${ }^{10-12}$

Secondary malignant cervical involvement is very unusual, and this may be due to cervical lymphatic flow towards the pelvic lymph nodes. ${ }^{1,2}$ Sometimes, this cervical lymphatic drainage is impaired due to previous abdominal surgery and/or massive metastasis to pelvic lymph nodes and this leads to secondary cervical involvement. Secondary cervical involvements are always associated with widespread and advanced stage of disease including pelvic lymph nodes, thus justifying the above hypothesis. $^{8-9}$

Cervical, vaginal or vulval metastasis are rare in case of ovarian tumour and rarely metastasis at these sites present in initial presentation of ovarian tumor. In a review study of 148 patients with FIGO stage III or IV ovarian cancer, 7 patients had cervical metastatic deposits. Four were due to direct extension of the primary; the 3 patients who had truly metastatic deposits all presented with cervical cytology showing the presence of adenocarcinoma cells. All the patients with cervical metastases had associated malignant ascites, retroperitoneal lymph node involvement, and significant peritoneal carcinomatosis. Their median survival was 4.4 months. $^{8}$

A large case series systemic review on adenocarcinoma metastasising to uterine cervix was done by PubMed search between the years 1970 and 2009 of case reports and series of cases of patients with metastatic gastric, breast, ovarian and colorectal cancer. Only 10 cases of tumours metastatic to the uterine cervix found. The study included the following reports in the systematic review: 13 reports of gastric-associated cancer, 30 related to breast cancer, nine with ovarian-associated cancer and 10 related to colorectal cancer. Thus, concluded that metastatic cervical activity is an infrequent event. ${ }^{13}$

Secondary metastatic lesions in cervix are multifocal or diffuse in nature. Presence of koilocytosis or cervical adenocarcinoma in situ in histopathological study almost always indicates the primary cervical nature of neoplasm. Association of HPV infection (in situ hybridization or
PCR) with above, adds in diagnosis of primary cervical involvement. P16 is expressed in many types of human malignancies, hence is less useful. ${ }^{14}$ Immunohistochemistry along with histopathology, also adds in diagnosis of secondary cervical metastasis. Immunohistochemical staining for CD34, CD31, and podoplanin are helpful. ${ }^{9}$

A single-institution study of 144 cases of secondary cervical malignancy, taking in account both histopathological and immunohistochemical criteria for correct diagnosis, concluded that secondary cervical malignancies present with wide spectrum of symptoms and almost invariably cervical metastasis indirectly indicates multiorgan involvement. ${ }^{9}$

The current standard treatment for patients with advanced ovarian cancer is primary cytoreductive surgery followed by chemotherapy. ${ }^{15}$ Neoadjuvant chemotherapy represents a few cycles of chemotherapy given prior to tumor cytoreductive surgery. It is suitable for patients with advanced ovarian cancer patients with generally poor quality, large tumor load, very high serum CA-125 level or tumor difficult to clean. ${ }^{16}$

In a retrospective study, patients with primarily unresectable ovarian cancer received a median of four platinum-based neoadjuvant chemotherapy courses, followed by surgery and adjuvant chemotherapy in patients with an objective response or stable disease after induction of chemotherapy. Neoadjuvant chemotherapy improves patients general condition, reduction of pleural effusion in addition to improving the patient's general condition to tolerate the surgery, control the pleural effusion and improves cytoreductive surgery success rate. 17

\section{CONCLUSION}

Cervical metastasis from an ovarian tumor is very rare. Authors report this case of bilateral serous cystadenocarcinoma metastasized to posterior lip of cervix, most probably by hematogenous route. Usually ovarian cancer with cervical metastases are at advanced stage and have associated malignant ascites, retroperitoneal lymph node involvement, and significant peritoneal carcinomatosis. Present patient was also at advanced stage (FIGO Stage IV) at the time of presentation, had gross ascites, bilateral pleural effusion and significant carcinomatosis but no retroperitoneal lymphadenopathy.

Neoadjuvant chemotherapy completely cured the pleural effusion and ascites before cytoreductive surgery. The prognosis of survival is poor in the presence of ovarian cancer with cervical metastases and it may be due to advanced stage of disease. Irrespective multimodal treatment, 6 cycles of postoperative chemotherapy and good follow-up authors lost present patient 11 months after surgery. 


\section{ACKNOWLEDGMENTS}

The authors are thankful to Dr. Pritinanda Mishra, Associate Professor, Department of Pathology, All India Institute of Medical Sciences, Bhubaneswar, India for performing the histopathological and immunological examinations.

Funding: No funding sources

Conflict of interest: None declared

Ethical approval: Not required

\section{REFERENCES}

1. Lemoine NR, Hall PA. Epithelial tumors metastatic to the uterine cervix, A study of 33 cases and review of the literature. Cancer 1986;57:2002-5.

2. Mulvany NJ, Nirenberg A, Oster AG. Non-primary cervical adenocarcinomas. Pathol. 1996;28:293-7.

3. Mc Cluggage WG, Hurrell DP, Kennedy K. Metastatic carcinomas in the cervix mimicking primary cervical adenocarcinoma and adenocarcinoma in situ: report of a series of cases. Am J Surg Pathol. 2010;34:735-41.

4. Malpica A, Deavers MT. Ovarian low-grade serous carcinoma involving the cervix mimicking a cervical primary. Int J Gynecol Pathol. 2011;30:613-9.

5. Bogliolo S, Morotti M, Valenzano Menada M, Fulcheri E, Musizzano Y, Casabona F. Breast cancer with synchronous massive metastasis in the uterine cervix: a case report and review of the literature. Arch Gynecol Obstet. 2010;281:769-73.

6. Green AE, Biscotti C, Michener C, Belinson J. Isolated cervical metastasis of breast cancer: a case report and review of the literature. Gynecol Oncol. 2004;95 267-9.

7. Kai K, Takai N, Nasu K, Kira N, Ishii T, Kashima K, et al. Metastatic uterine cervical cancer originating in the lung: a case report. Gynecol Obstet Invest. 2009;68:269-71.

8. Guidozzi F, Sonnendecker EW, Wright C. Ovarian cancer with metastatic deposits in the cervix, vagina, or vulva preceding cytoreductive surgery. Gynecol Oncol. 1993;49(2):225-8.

9. Zannoni GF, Vellone VG, Petrillo M. Secondary malignancies of the uterine cervix: a potential diagnostic pitfall. Virchows Arch. 2013;463(1):23-9.

10. Kawamoto S, Urban BA, Fishman EK. CT of epithelial ovarian tumors. Radiographics. 1999;19:S85-102.

11. Gourtsoyiannis N. Clinical MRI of the Abdomen, Why, How, When. Springer Verlag. 2011;ISBN:3540856889.

12. Gupta G, Massague J. Cancer metastasis: building a framework. Cell. 2006;127:679-95.

13. Pérez-Montiel D, Serrano-Olvera A. Adenocarcinoma metastatic to the uterine cevix; a case series. J Obstet Gynaecol Res. 2012;38(3):5419.

14. Witkiewicz AK, Knudsen KE, Dicker AP, Knudsen ES. The meaning of p16 (ink4a) expression in tumors: functional significance, clinical associations and future developments. Cell Cycle. 2011;10:2497503.

15. Monk BJ, Dalton H, Farley JH. Antiangiogenic agents as a maintenance strategy for advanced epithelial ovarian cancer. Crit Rev Oncol Hematol. 2013;86:161-75.

16. Le T, Hopkins L, Faught W. The lack of significance of $\mathrm{Ca} 125$ response in epithelial ovarian cancer patients treated with neoadjuvant chemotherapy and delayed primary surgical debulking. Gynecol Oncol. 2007;105:712-5.

17. Mazzeo F, Berliere M, Kerger J. Neoadjuvant chemotherapy followed by surgery and adjuvant chemotherapy in patients with primarily unresectable, advanced-stage ovarian cancer. Gynecol Oncol. 2003;90:163-9.

Cite this article as: Mallick A, Jena SK, Kuanar D. Bilateral ovarian serous cystadenocarcinoma metastasizing to cervix: a rare case report. Int J Reprod Contracept Obstet Gynecol 2018;7:4789-93. 